\title{
Association between receptor interacting serine/threonine kinase 2 polymorphisms and gastric cancer susceptibility
}

\author{
MASAFUMI OTA ${ }^{1}$, TOMOMITSU TAHARA ${ }^{2}$, TOSHIMI OTSUKA ${ }^{1}$, \\ WU JING $^{1}$, TOMOE NOMURA ${ }^{1}$, RANJI HAYASHI ${ }^{1}$, TAKEO SHIMASAKI ${ }^{1}$, \\ MASAKATSU NAKAMURA ${ }^{1}$, TOMOYUKI SHIBATA ${ }^{2}$ and TOMIYASU ARISAWA ${ }^{1}$ \\ ${ }^{1}$ Department of Gastroenterology, Kanazawa Medical University, Uchinada-machi, Ishikawa 920-0293; \\ ${ }^{2}$ Department of Gastroenterology, Fujita Health University, Toyoake, Aichi 470-1192, Japan
}

Received November 13, 2016; Accepted November 10, 2017

DOI: $10.3892 / 01.2018 .7785$

\begin{abstract}
The present study aimed to investigate whether single nucleotide polymorphisms in receptor interacting serine/threonine kinase 2 (RIPK2), which encodes a component of the nucleotide binding oligomerization domain containing 2-RIP2 pathway, may compromise the innate immune response to Helicobacter pylori infection, leading to increased susceptibility to gastric cancer in the Japanese population. The present case control study investigated the associations between RIPK2 single nucleotide polymorphisms and gastric mucosal inflammation, atrophy and cancer susceptibility in 528 patients with gastric cancer and 697 patients without gastric malignancies on upper gastro-duodenal endoscopy. Overall, the RIPK2 rs16900627 minor allele was significantly associated with the susceptibility to gastric cancer [OR, 1.37; 95\% confidence interval (CI), 1.06-1.77; $\mathrm{P}=0.016]$, particularly of the intestinal type (OR, 1.53; 95\% CI, 1.13-2.07; P=0.0062). It was also significantly associated with gastric mucosal atrophy (OR, 1.83; 95\% CI, 1.14-2.93; $\mathrm{P}=0.011$ ). When assessing the severity of chronic gastritis using the updated Sydney system, the activity and inflammation scores, as well as atrophy and metaplasia scores, were significantly higher in rs16900627 minor allele carriers compared with wild-type homozygotes. In patients younger than 60 years old, the pepsinogen I/II ratio was significantly lower in rs16900627 minor allele carriers compared with wild-type homozygotes $(\mathrm{P}=0.037)$. The rs16900627 minor allele is associated with the severity of gastric mucosal inflammation and the development of gastric mucosal atrophy. Carriers of this allele may have an increased
\end{abstract}

Correspondence to: Professor Tomiyasu Arisawa, Department of Gastroenterology, Kanazawa Medical University, 1-1 Daigaku, Uchinada-machi, Ishikawa 920-0293, Japan

E-mail: tarisawa@kanazawa-med.ac.jp

Key words: receptor interacting serine/threonine kinase 2, genetic polymorphism, gastric cancer, atrophic gastritis, Helicobacter pylori infection risk for the development of gastric cancer, particularly of the intestinal type.

\section{Introduction}

Helicobacter pylori (H. pylori) infection is now accepted as a crucial event in the development of atrophic gastritis, and is implicated in the development of gastric carcinoma (1-3). Gastric cancer develops incrementally, beginning with chronic inflammation, and progressing to atrophic inflammation, intestinal metaplasia, dysplasia and finally, frank malignancy (4). Whereas the majority of infected individuals are asymptomatic, chronic $H$. pylori infection in susceptible individuals is associated with variable degrees of mucosal damage (4). As a result, only a small percentage of infected individuals develop gastric cancer. The clinical outcome appears to be determined by the interplay of bacterial virulence factors, host gastric mucosal components and the environment. More specifically, a previous study revealed that genetic variability affecting elements of the mucosal immune system pivotally influences the clinical course of $H$. pylori infection (5).

Nucleotide-binding oligomerization domain-containing protein 2 /caspase recruitment domain-containing protein 15 (NOD2/CARD15) is a member of the NOD-like receptor gene family. It functions as an intracellular receptor for bacterial lipopolysaccharide, and is involved in signal transduction leading to the activation of the nuclear transcription factor- $\mathrm{\kappa B}$ (NF-kB) (6). Dysregulation of NOD2 signaling is associated with the pathogenesis of numerous inflammatory disorders (7). Indeed, NOD2 mutations are related to the occurrence of chronic inflammation of the gastric mucosa associated with H. pylori infection, development of intestinal metaplasia and dysplasia and ultimately gastric cancer (6). Previously, it was reported that the H. pylori bacterial Cag pathogenicity island and a cooperative interaction between the Toll-like receptor 2 (TLR2) /NOD2 and NOD-like receptor pyrin domain containing 3 protein, regulate interleukin-1 $\beta$ production in H. pylori infected dendritic cells (8). Initiation of NOD2 signaling is mediated by receptor-interacting protein 2 (RIP2), and the RIP2 interaction with NOD2 enhances NF- $\mathrm{kB}$ activity, making it an important player in cellular immune response (9). One study suggested that the innate immune system, including 
the NOD2-RIP2 signaling pathway, is involved in the pathogenesis of gastric inflammation and the development of gastric cancer (10).

RIP2 is an intracellular serine/threonine kinase that contains a caspase recruitment domain at its carboxy terminus. Single nucleotide polymorphisms (SNPs) of the receptor interacting serine/threonine kinase 2 (RIPK2) gene, encoding RIP2, are associated with systemic lupus erythematosus (SLE) (11), and with the severity of childhood atopic asthma (12). However, although the association of NOD2 and TLR polymorphisms with gastric cancer susceptibility have been described, RIPK2 polymorphisms have not been studied in this context $(13,14)$.

The present study investigated the association between RIPK2 gene polymorphisms and gastric cancer susceptibility in a Japanese population. The present study also investigated the association between RIPK2 polymorphisms and the severity of chronic gastritis in subjects without gastric cancer.

\section{Materials and methods}

Study subjects. All patients with gastric cancer attended the Endoscopy Center of Kanazawa Medical University (Uchinada-machi, Japan) or Fujita Health University (Kutsukake-cho, Japan) from April 2005 to March 2015, and were diagnosed by endoscopy and pathological examination of biopsy samples. Gastric cancer was classified according to Lauren's classification (15). Non-cancer patients complaining of abdominal discomfort underwent endoscopic examination, and were diagnosed as having a gastric ulcer, duodenal ulcer, gastritis or no apparent gastric disease. Patients who had severe systemic disease and received non-steroidal anti-inflammatory drugs were excluded from the present study. Finally, the study population comprised 1,221 subjects whose polymorphisms could be clearly analyzed, including 524 patients with gastric cancer (GC group, mean age, 65.4 11.4 ; age range, 23-94; male/female, 371/133) and 697 subjects without gastric cancer (non-GC group; mean age, 60.9 \pm 13.6 ; age range, 22-93; male/female, 400/297). In 428/697 patients in the non-GC group (mean age, 60.0 \pm 13.3 ; age range, 26-93; male/female, 254/174), the severity of chronic gastritis was assessed using antral biopsy specimens and classified according to the updated Sydney system (16). All pathology assessment was performed by a pathologist at Fujita Health University Hospital who was blinded to any clinical information. According to the estimated histological gastritis scores, the atrophic gastritis group (AT group) was defined as atrophy score $\geq 2$ or metaplasia score $\geq 1$, and the others were classified into the non-atrophy group. In addition, serum pepsinogen (PG) I/II levels were evaluated in 134/428 subjects without gastric cancer. The Ethics Committees of Fujita Health University and Kanazawa Medical University approved the protocol. Written informed consent was obtained from all of the participating patients prior to enrollment in the present study.

SNP selection and detection. A significant association between the rs16900627 SNP and SLE susceptibility has been previously reported (11). The polymorphism is located in the 3 ' untranslated region of RIPK2, a region rich in microRNA binding sites, and is associated with other SNPs, including rs7844627, 10504881. In addition, rs2230801, encoding a non-synonymous substitution (Ile259Thr), was identified in RIPK2. The distribution of the two SNP genotypes were confirmed using the HapMap database (https://snpinfo .niehs.nih.gov/snpinfo/snptag.html) and National Centre for Biotechnology Information (NCBI) SNP database (http://www.ncbi.nlm.nih.gov/snp/) (17). The present study selected the rs16900627 and rs2230801 SNPs for further investigation on the basis that they may affect innate immune signaling. For genotype determination, the present study used polymerase chain reaction-single strand conformation polymorphism (PCR-SSCP) methods using DNA samples prepared from peripheral blood as reported previously (18). All PCRs were performed in a volume of $20 \mu \mathrm{l}$ containing $0.1 \mu \mathrm{g}$ of genomic DNA. Primer pairs used to detect rs16900627 were as follows: Forward, 5'-CTGATGGAAGCCATTTTCACA TTCAT-3' and reverse, 5'-TCTGTCTCTGGTGGGTAAAGG GTAT-3. The DNA was denatured at $95^{\circ} \mathrm{C}$ for $3 \mathrm{~min}$, followed by 35 cycles at $96^{\circ} \mathrm{C}$ for $15 \mathrm{sec}, 50^{\circ} \mathrm{C}$ for $40 \mathrm{sec}$ and $72^{\circ} \mathrm{C}$ for $30 \mathrm{sec}$, with a final extension at $72^{\circ} \mathrm{C}$ for $5 \mathrm{~min}$. Primer pairs used to detect rs2230801 were as follows: Forward, 5'-TCC TTTGCAGATAATGTATAGTGTGTCA-3' and reverse, 5'-AGAGATCATACGTGCTCGGTGAGGT-3'. The DNA was denatured at $95^{\circ} \mathrm{C}$ for $3 \mathrm{~min}$, followed by 35 cycles at $96^{\circ} \mathrm{C}$ for $15 \mathrm{sec}, 58^{\circ} \mathrm{C}$ for $40 \mathrm{sec}$ and $72^{\circ} \mathrm{C}$ for $30 \mathrm{sec}$, with final extension at $72^{\circ} \mathrm{C}$ for $5 \mathrm{~min}$. Subsequently, $2 \mu \mathrm{l}$ of both PCR products was denatured with $10 \mu \mathrm{l}$ formamide (Sigma-Aldrich; Merck KGaA, Darmstadt, Germany) at $90^{\circ} \mathrm{C}$ for $5 \mathrm{~min}$. SSCP was performed at $18^{\circ} \mathrm{C}$ using a GenePhor DNA separation system with GeneGel Excel 12.5/24 (GE Healthcare, Chicago, IL, USA), after which the denatured single strand DNA was stained using a DNA Silver Staining kit (GE Healthcare) according to the manufacturer's protocol.

Statistical analysis. Hardy-Weinberg equilibrium was assessed by $\chi^{2}$ statistics. The age data are presented as the mean \pm standard deviation. Mean ages between two groups was compared by Student's t-test. The ratios of $H$. pylori infection status and gender were compared by Fisher's exact test. Differences of genotype frequencies were determined by two-sided Fisher's exact test. The odds ratios (OR) and 95\% confidence intervals (CI) were also determined by logistic regression with adjustment for age, sex and $H$.pylori infection status. The Sydney system scores and PG I/II ratio between two groups were compared by Mann Whitney U-test. $\mathrm{P}<0.05$ was considered to indicate a statistically significant difference. Analyses were performed using STATA Version 13 (StataCorp LP, College Station, TX, USA).

\section{Results}

Characteristics of the study subjects and the frequencies of genotypes. The characteristics of subjects in the present study are summarized in Table I. The mean age, male:female ratio and $H$. pylori positivity of the GC group were significantly higher compared with those of the non-GC group. Single strand DNAs of rs16900627 and rs2230801 were clearly separated by SSCP (Fig. 1). The distribution of the rs16900627 genotype in 697 subjects without gastric cancer was 554AA, 130AG and $13 \mathrm{GG}$ (Table I), and that of rs2230801 was 681TT and 16TC. The CC genotype of rs2230801 was not identified. The 
Table I. Characteristics of the subjects and frequencies of genotypes.

\begin{tabular}{lccc}
\hline Characteristic & Non-GC group & GC group & P-value \\
\hline No. of subjects & 697 & 524 & \\
Mean age \pm SD & $60.9 \pm 13.6$ & $65.4 \pm 11.4$ & $<0.001$ \\
Male:female & $400: 297$ & $371: 133$ & $<0.001$ \\
H. pylori positivity & $430 / 697$ & $457 / 524$ & $<0.001$ \\
rs16900627 & & & \\
$(* 351 \mathrm{~A}>\mathrm{G})$ & & & \\
AA & 554 & 395 & 0.029 \\
AG & 130 & 115 & \\
GG & 13 & 14 & 0.432 \\
G allele frequency & $11.2 \%$ & $13.6 \%$ & 0.071 \\
rs2230801 & & & \\
(776T>C, Ile259Thr) & & & \\
TT & 681 & 517 & 0.289 \\
TC & 16 & 7 & \\
CC & 0 & 0 & \\
C allele frequency & $1.15 \%$ & $0.67 \%$ & 0.291 \\
\hline
\end{tabular}

$\mathrm{SD}$, standard deviation; GC, gastric cancer.
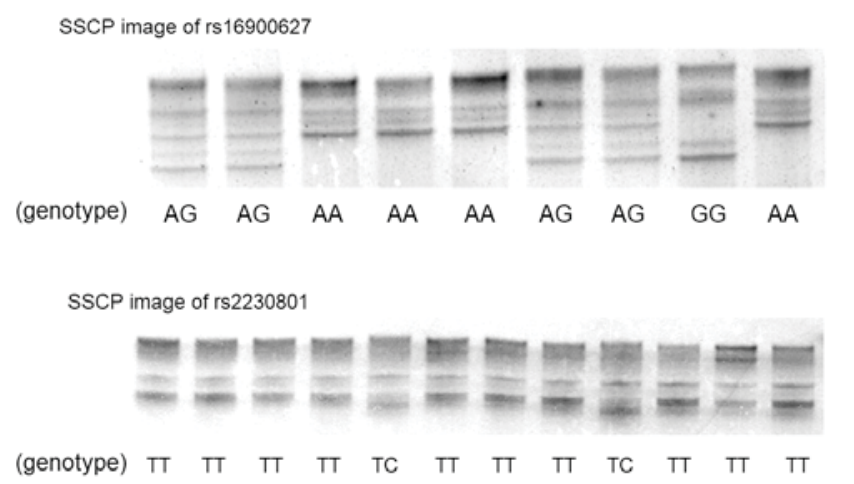

Figure 1. Polymerase chain reaction-SSCP images using clinical samples. Single strand DNAs were separated by SSCP. The genotype could be determined. SSCP, single strand conformation polymorphism.

distribution of genotypes was in Hardy-Weinberg equilibrium (rs16900627 and rs2230801; $\mathrm{P}=0.12$ and $\mathrm{P}=1.0$, respectively). The wild-type rs16900627 homozygote frequency was significantly lower in the GC group compared with in the non-GC group $(\mathrm{P}=0.029)$, whereas the frequencies of other genotypes were not significantly different (Table I).

Association between gene polymorphisms and gastric cancer susceptibility. By logistic regression analysis following adjustment for age, sex and H.pylori infection status, the rs16900627 AG+GG genotypes were revealed to be significantly associated with susceptibility to gastric cancer (OR, 1.38; 95\% CI, 1.03-1.84; $\mathrm{P}=0.032$; Table II), and more strongly associated with the intestinal type of gastric cancer (OR, 1.56; $95 \%$ $\mathrm{CI}, 1.11-2.20 ; \mathrm{P}=0.011)$. In addition, if the number of minor alleles was considered to be a co-variable $(\mathrm{GG}=2, \mathrm{AG}=1$ and
$\mathrm{AA}=0$ ), it was also significantly associated with gastric cancer susceptibility (OR, 1.37; 95\% CI, 1.06-1.77; $\mathrm{P}=0.016$ ), particularly with the intestinal type (OR, 1.53; 95\% CI, 1.13-2.07; $\mathrm{P}=0.0062$; Table II). Conversely, the rs2230801 genotype was not significantly associated with gastric cancer susceptibility (Table III).

Association between gene polymorphisms and severity of chronic gastritis. The characteristics of 428 subjects without gastric cancer whose histological severity was assessed are presented in Table IV. The genotype distribution in this sample was not significantly different from that of the non-GC group (rs16900627 and rs2230801; $\mathrm{P}=0.11$ and $\mathrm{P}=1.0$, respectively). As the mean values of atrophy and metaplasia scores were 1.13 and 0.652 , respectively, the AT group was defined as atrophy score $\geq 2$ or metaplasia score $\geq 1$. On this basis, mean age, male:female ratio and $H$. pylori positivity in the AT group were significantly higher compared with those in the non-AT group. Furthermore, the frequency of the rs16900627 AA genotype was significantly lower, and the minor allele frequency was also significantly higher in the AT group $(\mathrm{P}=0.011$ and $\mathrm{P}=0.0060$, respectively). By logistic regression analysis, the rs16900627 minor allele carriers had a significantly increased risk for gastric mucosal atrophy (OR, 1.72; 95\% CI, 1.01-2.96; $\mathrm{P}=0.048$ ) and the significance was increased when the number of minor alleles was set as a co-variable (OR, 1.83; 95\% CI, 1.14-2.93; P=0.011, Table V). Conversely, there was no significant association between rs2230801 and severity of gastric mucosal atrophy. In addition, all Sydney system scores were significantly higher in rs16900627 minor allele carriers compared with in AA homozygotes (Fig. 2). The rs16900627 genotype distribution in 134 subjects whose serum pepsinogens were determined was $102 \mathrm{AA}, 28 \mathrm{AG}$ and $4 \mathrm{GG}$, which was not significantly different from that in the non-GC group $(\mathrm{P}=0.56)$. Overall, there was no significant difference in $\mathrm{PG}$ I/II ratio between rs16900627 AA homozygotes and minor allele carriers (Fig. 3). However, in subjects younger than 60 years, the ratio was significantly lower in minor allele carriers compared with in AA homozygotes $(\mathrm{P}=0.037)$.

\section{Discussion}

The immune system is composed of innate and adaptive parts, and $H$. pylori infection induces both parts of the host immune response (19). Recently, the innate immune response to $H$. pylori was revealed to be an important factor affecting gastric mucosal inflammation $(8,20)$. The present study previously reported a significant association between the TLR2-196 to -174 deletion polymorphism and gastric cancer susceptibility in Japanese patients (21), which was consistent with other Japanese studies associating TLR gene polymorphisms to gastric cancer (14). NOD2 polymorphisms are also associated with changes in gastric mucosa, which lead to gastric cancer susceptibility $(13,22,23)$. This suggested that genetic variations affecting the innate immune response may impact gastric cancer susceptibility. RIP2 is involved in both innate and adaptive parts of the immune response (24). Therefore, $R I P K 2$ genetic variation may influence the severity of gastric mucosal inflammation, the progression of gastric atrophy and the development of gastric cancer. 
Table II. Association between rs16900627 polymorphisms and gastric cancer.

A, G allele carriers vs. the others

\begin{tabular}{lrrrrr}
\hline rs16900627 & AA & AG & GG & AG+GG vs. AA, OR (95\% CI) \\
\hline Non-GC (697) & 554 & 130 & 13 & Reference \\
Overall GC (524) & 395 & 115 & 14 & $1.38(1.03-1.84)$ & $1.56(1.11-2.20)$ \\
Intestinal (309) & 227 & 74 & 8 & $1.15(0.778-1.69)$ & 0.032 \\
Diffuse (212) & 166 & 40 & 6 & - & 0.011 \\
Unknown & 2 & 1 & 0 & - \\
\hline
\end{tabular}

B, Co-variable: The number of G allele

\begin{tabular}{lrrrcr}
\hline rs16900627 & AA & AG & GG & No. of G allele, OR (95\% CI) \\
\hline Non-GC (697) & 554 & 130 & 13 & Reference \\
Overall GC (524) & 395 & 115 & 14 & $1.37(1.06-1.77)$ & 0.016 \\
Intestinal (309) & 227 & 74 & 8 & $1.53(1.13-2.07)$ & 0.006 \\
Diffuse (212) & 166 & 40 & 6 & $1.20(0.857-1.68)$ & - \\
Unknown & 2 & 1 & 0 & - & -
\end{tabular}

${ }^{a}$ The number of subjects following logistic regression analysis after adjustment for age, gender and $H$. pylori infection status. CI, confidence interval; GC, gastric cancer; OR, odds ratio.

Table III. Association between rs2230801 polymorphisms and gastric cancer.

\begin{tabular}{|c|c|c|c|c|c|}
\hline rs $2230801^{\mathrm{a}}$ & TT & $\mathrm{TC}$ & $\mathrm{CC}$ & TC vs. TT, OR (95\% CI) & P-value \\
\hline Non-GC (697) & 681 & 16 & 0 & Reference & \\
\hline Overall GC (524) & 517 & 7 & 0 & $0.531(0.210-1.34)$ & 0.18 \\
\hline Intestinal (309) & 304 & 5 & 0 & $0.664(0.229-1.92)$ & 0.45 \\
\hline Diffuse (212) & 210 & 2 & 0 & $0.344(0.077-1.53)$ & 0.16 \\
\hline Unknown & 3 & 0 & 0 & - & - \\
\hline
\end{tabular}

${ }^{a}$ The number of subjects following logistic regression analysis after adjustment for age, gender and $H$. pylori infection status. CI, confidence interval; GC, gastric cancer; OR, odds ratio.

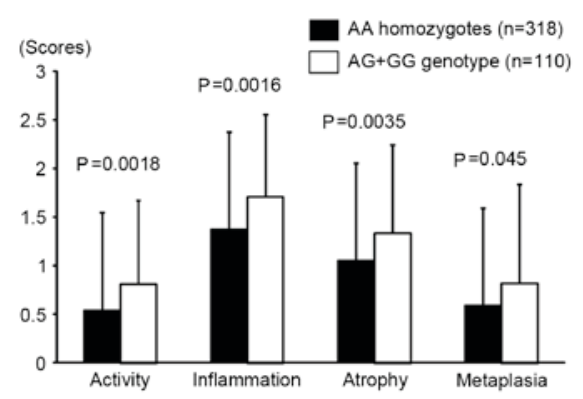

Figure 2. Comparison of each updated Sydney system score among rs16900627 AA homozygotes and AG+GG genotypes. All of the updated Sydney system scores were significantly higher in the AG+GG genotype than the AA homozygotes.

The results of the present study provide the first evidence, to the best of our knowledge, that RIPK2 genetic polymorphisms are significantly associated with susceptibility to gastric cancer in the Japanese population. The rs16900627 A $>\mathrm{G}$ minor allele was associated with an increased risk for the development of gastric cancer, particularly the intestinal type. The frequency of rs16900627 in the Japanese population is reported in the HapMap database. However, in the present study the distribution of the genotype was different from the controls without gastric cancer $(\mathrm{P}=0.045)$, even though the genotype distribution in the control subjects was in Hardy-Weinberg equilibrium. Conversely, the genotype distribution of 232 subjects without histological gastric mucosal atrophy was the same as that in the HapMap database $(\mathrm{P}=0.13)$. This discrepancy may be explained by the fact that all of the control subjects included were patients who had an endoscopic examination due to various symptoms, including abdominal symptoms and abnormal findings revealed by a health check. The frequency of rs2230801 is not reported in HapMap, but the genotype distribution was in the Hardy-Weinberg equilibrium in the control subjects. 
Table IV. Characteristics and genotype frequencies in the subjects that underwent severity of gastritis evaluation.

\begin{tabular}{|c|c|c|c|c|}
\hline Characteristic & Total & Non-AT group & AT group & P-value \\
\hline No. of subjects & 428 & 232 & 196 & \\
\hline Mean age $\pm \mathrm{SD}$ & $60.0 \pm 13.3$ & $57.8 \pm 14.2$ & $62.5 \pm 11.6$ & $<0.001$ \\
\hline Male: Female & $254: 174$ & 123:109 & $131: 65$ & 0.004 \\
\hline H. pylori positive rate & $278 / 428$ & $94 / 232$ & $184 / 196$ & $<0.001$ \\
\hline \multicolumn{5}{|l|}{ rs16900627 A>G } \\
\hline AA & 318 & 184 & 134 & 0.011 \\
\hline $\mathrm{AG}$ & 98 & 44 & 54 & \\
\hline GG & 12 & 4 & 8 & \\
\hline $\mathrm{G}$ allele frequency & $14.1 \%$ & $11.2 \%$ & $17.9 \%$ & 0.006 \\
\hline \multicolumn{5}{|l|}{ rs2230801 T>C } \\
\hline TT & 416 & 228 & 188 & \\
\hline $\mathrm{TC}$ & 12 & 4 & 8 & \\
\hline $\mathrm{CC}$ & 0 & 0 & 0 & \\
\hline $\mathrm{C}$ allele frequency & $1.40 \%$ & $0.86 \%$ & $2.04 \%$ & \\
\hline
\end{tabular}

AT, atrophic gastritis.

Table V. Association between RIPK2 polymorphisms and gastric mucosal atrophy.

A, rs $16900627, \mathrm{G}$ allele carriers vs. the others

\begin{tabular}{lcccc}
\hline rs16900627 & AA & AG & GG & AG+GG vs. AA, OR (95\% CI) \\
\hline Non-AT group (232) & 184 & 44 & 4 & Reference \\
AT group (196) & 134 & 54 & 8 & $1.72(1.01-2.96)$ \\
\hline
\end{tabular}

rs16900627, co-variable: the number of G allele

\begin{tabular}{lcccc}
\hline B, rs16900627 & AA & AG & GG & No. of G allele, OR (95\% CI) \\
\hline Non-AT group (232) & 184 & 44 & 4 & Reference \\
AT group (196) & 134 & 54 & 8 & $1.83(1.14-2.93)$ \\
\hline
\end{tabular}

C, rs2230801, TC vs. TT

\begin{tabular}{llcccc}
\hline rs2230801 & TT & TC & CC & TC vs. TT, OR (95\% CI) \\
\hline Non-AT group (232) & 228 & 4 & 0 & Reference \\
AT group (196) & 188 & 8 & 0 & $1.70(0.441-6.99)$ & 0.472
\end{tabular}

${ }^{a}$ The number of subjects following logistic regression analysis after adjustment for age, gender and $H$. pylori infection status. RIPK2, receptor interacting serine/threonine kinase 2; CI, confidence interval; OR, odds ratio; AT, atrophic gastritis.

To date, there have been few studies associating RIPK2 genetic variations and clinical disease susceptibility $(11,12,25)$. Li et al (11) reported that the rs 16900627 minor allele was significantly associated with SLE susceptibility. This suggested that the mutant rs16900627 genotype may be associated with an increased risk of chronic inflammation via an alteration of innate immune responses, although the detailed mechanisms remain unclear. Similarly, in the present study, activity and inflammation scores based on the Sydney system were higher in $\mathrm{AG}+\mathrm{GG}$ genotypes compared with in AA homozygotes, although $H$. pylori positivity was not significantly different $(67.3$ and $64.1 \%$, respectively). Atrophy and metaplasia scores were also significantly higher in AG+GG genotypes compared with in 


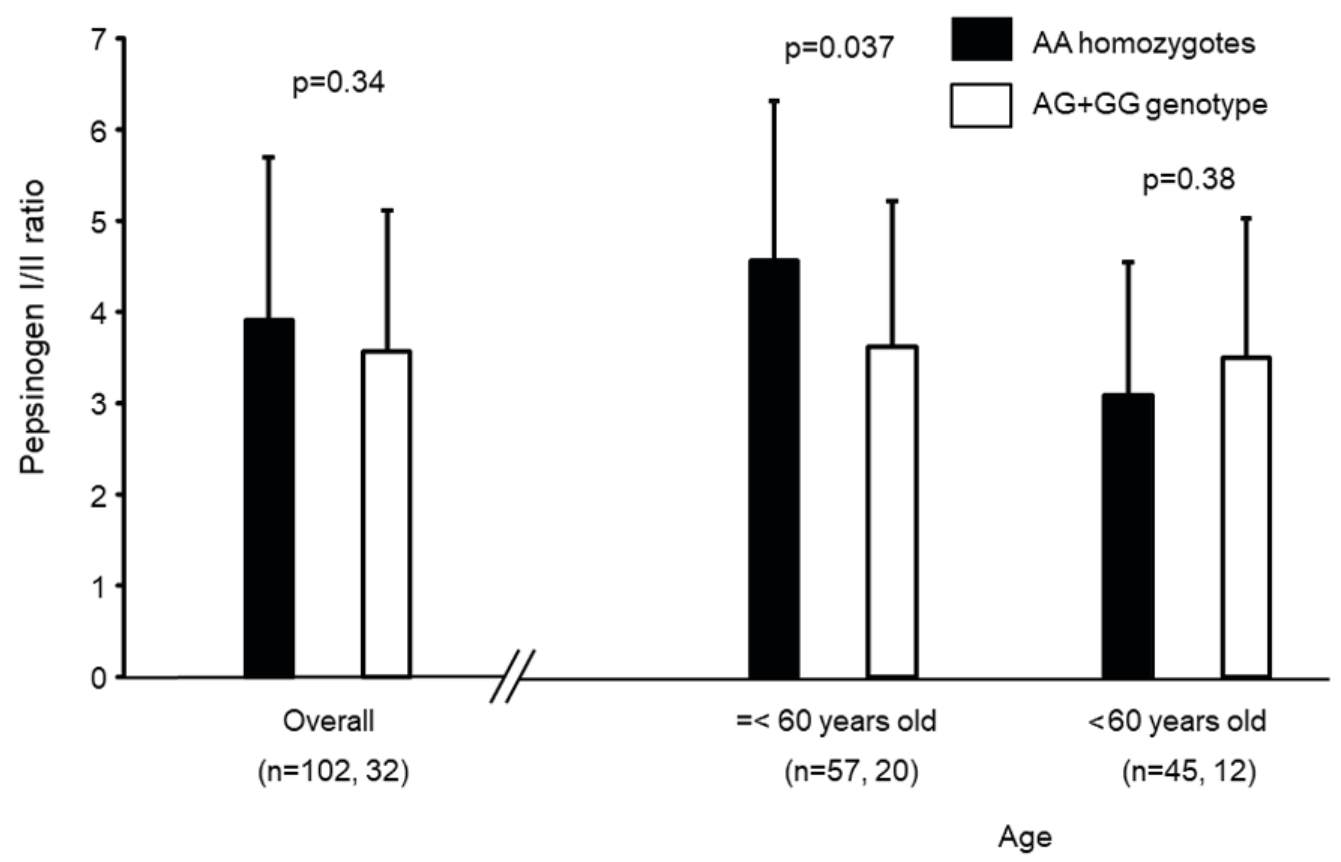

Figure 3. The serum PG I/II ratio and rs16900627 A>G genotype. Overall, serum PG I/II ratio did not differ between AA homozygotes and AG+GG genotypes. However, in the subjects younger than 60 years, it was significantly lower in the AG+GG genotypes compared with in the AA homozygotes. PG, pepsinogen.

AA homozygotes, and logistic regression analysis indicated that the rs160900627 minor allele genotype was associated with an increased risk with the progression of gastric mucosal atrophy.

However, by serological examination, significant differences of PG I/II ratio between AG+GG genotypes and AA homozygotes were not observed in older patients (age $>60$ years old), although it was significantly different in younger age groups (age $\leq 60$ years old). Sydney system scores express the severity of gastritis at a biopsy point, whereas PG I/II ratio express the spread of mucosal atrophy $(16,26)$. It has been reported that the significant association between serum and histological atrophy is more strongly observed in the corpus compared with in the antrum (27). The present study assessed the histological gastritis only in the antrum, as the antrum is affected by $H$. pylori infection for the longest period (4). This may be the reason for the difference of results between histological and serum atrophy. The present study suggested that the results of PG I/II ratio indicated that the mucosal atrophy progressed more extensively in $\mathrm{AG}+\mathrm{GG}$ genotypes compared with in AA homozygotes at an earlier stage of $H$. pylori infection, and that the difference of the value of PG I/II ratio between the two groups may be obscured by the mucosal atrophy spreading following a long period. In addition, when the comparatively large deviation of the PG I/II ration was considered, the sample size of the present study may be too small.

The results of the present study demonstrated a significant association between the rs16900627 minor genotype and the intestinal type of gastric cancer. The intestinal type consists of gland-like structures that mimic the intestinal glands, and a series of precancerous intestinal type lesions are recognized, beginning with chronic inflammation of the stomach and passing through the intermediate stages of atrophic gastritis or intestinal metaplasia (28). The results of the present study suggested that the rs16900627 minor allele genotype may be associated with the severity of gastric mucosal atrophy, and may increase the risk for developing gastric mucosal atrophy associated disorders, including intestinal type gastric cancer.

There was no significant association between rs2230801, and gastric mucosal atrophy and gastric cancer susceptibility. This polymorphism causes an Ile-to-Thr substitution at amino acid 259, but is a minor variant in the NCBI SNP database (http://www.ncbi.nlm.nih.gov/snp/). Furthermore, there was no minor allele homozygote in the present study. Overall, these findings suggested that this polymorphism had little, if any, association with gastric disorders.

There were certain clinical limitations to the present study. The present study included patients who visited the Fujita Health University Hospital to undergo endoscopic examination due to specific symptoms or further checks after a general health check. The subjects who had no symptoms were included in the control group. In addition, endoscopy is limited in its ability to detect small histological neoplasia. The present study was not able to confirm whether very small histological neoplasia were present in the control group. Another limitation was that the present study assessed histological gastritis using biopsy samples only from the antrum. Further results may be provided if the severity of gastritis in the corpus was assessed at the same time. The main disadvantage was the relatively small sample size used in the present study, particularly with respect to the number of subjects that had their serum pepsinogen levels assessed. Due to the relatively large deviation of pepsinogen levels, a greater sample size will be required to assess the PG I/II ratio. The final limitation of the study design was that only using samples stored in a single center were analyzed retrospectively.

In conclusion, the results of the present study suggest that the rs16900627 minor allele is associated with the severity of gastric mucosal inflammation and the development of gastric 
mucosal atrophy, and carriers of this allele may have an increased risk for the development of gastric cancer, particularly of the intestinal type. These results will be useful in predicting which $H$.pylori infected patients will be at a higher risk of developing gastric cancer.

\section{References}

1. Blaser MJ and Parsonnet J: Parasitism by the 'slow' bacterium Helicobacter pylori leads to altered gastric homeostasis and neoplasia. J Clin Invest 94: 4-8, 1994.

2. Huang JQ, Sridhar S, Chen Y and Hunt RH: Meta-analysis of the relationship between Helicobacter pylori seropositivity and gastric cancer. Gastroenterology 114: $1169-1179,1998$

3. Uemura N, Okamoto S, Yamamoto S, Matsumura $N$ Yamaguchi S, Yamakido M, Taniyama K, Sasaki N and Schlemper RJ: Helicobacter pylori infection and the development of gastric cancer. N Engl J Med 345: 784-789, 2001.

4. Correa P: Human gastric carcinogenesis: A multistep and multifactorial process-First American Cancer Society Award Lecture on Cancer Epidemiology and Prevention. Cancer Res 52: 6735-6740, 1992.

5. Crabtree JE: Gastric mucosal inflammatory responses to Helicobacter pylori. Aliment Pharmacol Ther 10 (Suppl 1): S29-S37, 1996.

6. Rosenstiel P, Hellmig S, Hampe J, Ott S, Till A, Fischbach W, Sahly H, Lucius R, Fölsch UR, Philpott D and Schreiber S: Influence of polymorphisms in the NOD1/CARD4 and NOD2/CARD15 genes on the clinical outcome of Helicobacter pylori infection. Cell Microbiol 8: 1188-1198, 2006.

7. Tigno-Aranjuez JT and Abbott DW: Ubiquitination and phosphorylation in the regulation of NOD2 signaling and NOD2-mediated disease. Biochim Biophys Acta 1823: 2022-2028, 2012.

8. Kim DJ, Park JH, Franchi L, Backert S and Núñez G: The Cag pathogenicity island and cooperative interaction between TLR2/NOD2 and NLRP3 regulate IL-1 $\beta$ production in Helicobacter pylori-infected dendritic cells. Eur J Immunol 43: 2650-2658, 2013.

9. Ogura Y, Inohara N, Benito A, Chen FF, Yamaoka S and Nunez G: Nod2, a Nod1/Apaf-1 family member that is restricted to monocytes and activates NF-kappaB. J Biol Chem 276: 4812-4818, 2001

10. Li ZX, Wang YM, Tang FB, Zhang L, Zhang Y, Ma JL, Zhou T, You WC and Pan KF: NOD1 and NOD2 genetic variants in association with risk of gastric cancer and its precursors in a Chinese population. PLoS One 10: e0124949, 2015.

11. Li J, Tian J, Ma Y, Cen H, Leng RX, Lu MM, Chen GM, Feng CC, Tao JH, Pan HF and Ye DQ: Association of RIP2 gene polymorphisms and systemic lupus erythematosus in a Chinese population. Mutagenesis 27: 319-322, 2012.

12. Nakashima K, Hirota T, Suzuki Y, Akahoshi M, Shimizu M, Jodo A, Doi S, Fujita K, Ebisawa M, Yoshihara S, et al: Association of the RIP2 gene with childhood atopic asthma. Allergol Int 55: 77-83, 2006.

13. Liu J, He C, Xu Q, Xing C and Yuan Y: NOD2 polymorphisms associated with cancer risk: A meta-analysis. PLoS One 9: e89340, 2014.
14. Castaño-Rodríguez N, Kaakoush NO, Goh KL, Fock KM and Mitchell HM: The role of TLR2, TLR4 and CD14 genetic polymorphisms in gastric carcinogenesis: A case-control study and meta-analysis. PLoS One 8: e60327, 2013.

15. Lauren P: The two histological main types of gastric carcinoma: Diffuse and so-called intestinal-type carcinoma. An attempt at a histo-clinical classification. Acta Pathol Microbiol Scand 64: 31-49, 1965.

16. Dixon MF, Genta RM, Yardley JH and Correa P. Classification and grading of gastritis. The updated sydney system. International Workshop on the Histopathology of Gastritis, Houston 1994. Am J Surg Pathol 20: 1161-1181, 1996.

17. Sherry ST, Ward MH, Kholodov M, Baker J, Phan L, Smigielski EM and Sirotkin K: dbSNP: The NCBI database of genetic variation. Nucleic Acids Res 29: 308-311, 2001.

18. Arisawa T, Tahara T, Ozaki K, Matsue Y, Minato T, Yamada H, Nomura T, Hayashi R, Matsunaga K, Fukumura A, et al: Association between common genetic variant of $\mathrm{HRH} 2$ and gastric cancer risk. Int J Oncol 41: 497-503, 2012.

19. Algood HM and Cover TL: Helicobacter pylori persistence: An overview of interactions between $\mathrm{H}$. pylori and host immune defenses. Clin Microbiol Rev 19: 597-613, 2006.

20. Rad R, Ballhorn W, Voland P, Eisenächer K, Mages J, Rad L, Ferstl R, Lang R, Wagner H, Schmid RM, et al: Extracellular and intracellular pattern recognition receptors cooperate in the recognition of Helicobacter pylori. Gastroenterology 136: 2247-2257, 2009.

21. Tahara T, Arisawa T, Wang F, Shibata T, Nakamura M, Sakata M, Hirata I and Nakano H: Toll-like receptor $2-196$ to 174del polymorphism influences the susceptibility of Japanese people to gastric cancer. Cancer Sci 98: 1790-1794, 2007.

22. Rigoli L, Di Bella C, Fedele F, Procopio V, Amorini M, Lo Giudice G, Romeo P, Pugliatti F, Finocchiaro G, Lucianò R and Caruso RA: TLR4 and NOD2/CARD15 genetic polymorphisms and their possible role in gastric carcinogenesis. Anticancer Res 30: 513-517, 2010.

23. Hnatyszyn A, Szalata M, Stanczyk J, Cichy W and Slomski R: Association of c. $802 \mathrm{C}>\mathrm{T}$ polymorphism of NOD2/CARD15 gene with the chronic gastritis and predisposition to cancer in H. pylori infected patients. Exp Mol Pathol 88: 388-393, 2010

24. Watanabe T, Asano N, Fichtner-Feigl S, Gorelick PL, Tsuji Y, Matsumoto Y, Chiba T, Fuss IJ, Kitani A and Strober W: NOD1 contributes to mouse host defense against Helicobacter pylori via induction of type I IFN and activation of the ISGF3 signaling pathway. J Clin Invest 120: 1645-1662, 2010.

25. Marcinek P, Jha AN, Shinde V, Sundaramoorthy A, Rajkumar R, Suryadevara NC, Neela SK, van Tong H, Balachander V, Valluri VL, et al: LRRK2 and RIPK2 variants in the NOD 2-mediated signaling pathway are associated with susceptibility to Mycobacterium leprae in Indian populations. PLoS One 8: e73103, 2013

26. Huang YK, Yu JC, Kang WM, Ma ZQ, Ye X, Tian SB and Yan C: Significance of serum pepsinogens as a biomarker for gastric cancer and atrophic gastritis screening: A systematic review and meta-analysis. PLoS One 10: e0142080, 2015.

27. Lee JY, Kim N, Lee HS, Oh JC, Kwon YH, Choi YJ, Yoon KC, Hwang JJ, Lee HJ, Lee A, et al: Correlations among endoscopic, histologic and serologic diagnoses for the assessment of atrophic gastritis. J Cancer Prev 19: 47-55, 2014.

28. Go MF: Review article: Natural history and epidemiology of Helicobacter pylori infection. Aliment Pharmacol Ther 16 (Suppl 1): S3-S15, 2002. 